\title{
SYMMETRY TESTS WITH HIGHLY CHARGED IONS *
}

\author{
Robert R. LEWIS \\ Department of Physics, University of Michigan, Ann Arbor, MI 48109, USA
}

\begin{abstract}
We summarize the present status of testing the standard model of parity nonconservation in neutral atoms, emphasizing the success in understanding the semileptonic weak interactions. There is good agreement for the nuclear spin independent contributions, especially in cesium. There is some evidence of nuclear spin dependent terms, probably due to hadronic weak interactions. There is no evidence of leptonic weak interactions. The lessons learned from atomic physics are discussed, together with strategies for parity experiments using highly charged ions.
\end{abstract}

\section{Introduction}

As far as I can ascertain, the subject I was urged to discuss is nonexistent: there have been no symmetry tests in highly charged ions and there are no experiments underway. Many people, some of them present, have considered this situation and concluded that symmetry tests with highly charged ions have no advantages over those with neutral atoms. Nevertheless, it seems appropriate at a workshop like this to discuss ideas and guidelines for the future. Perhaps someone here will see the lack of results as an opportunity, and will find a way of adding to this "null topic".

My talk begins with a short review of the status of parity tests in atomic physics, summarizing the status of what has been learned so far. Nothing will be included on the subject of time reversal tests, since these effects are known to be at most "micro-weak". Instead, I will emphasize the problems of testing the standard model, which makes many definite predictions. The material progresses from the most precise to the least, and tries to clarify what features are inaccessible to experiments with atoms. Time does not permit a complete review; I just pick a few good examples. Then I will try to discuss what the experience with atoms has to teach about the design of parity experiments. The talk will end with a brief survey of proposals for hydrogen-like and heliumlike ions, and a discussion of strategies based on the experience with atoms.

\section{Parity tests in heavy atoms}

The standard model of electroweak interactions

* Invited presentation at the Workshop on Highly Charged Ions: New Physics and Advanced Techniques, Berkeley, CA, USA, March 13-15, 1989. makes a definite prediction of the breaking of parity conservation in the semileptonic interaction between the electrons and nucleons [1]. Hadronic interactions between the nucleons and leptonic interactions between the electrons are also predicted. Moreover, these predictions lead to observable consequences in atomic physics [2] which can be compared with results from high energy physics. The model has only one adjustable parameter, the Weinberg angle $\theta_{\mathrm{W}}$, which appears in the coupling strengths. There are two types of semileptonic interactions, resulting from the exchange of a $Z^{0}$ between the electrons and nucleus. The first $\left(\mathscr{H}_{\text {VA }}\right)$ comes from the product of the hadronic vector current with the electronic axial current and has coupling constants called $C_{1}$. The second $\left(\mathscr{H}_{\text {AV }}\right)$ comes from the hadronic axial current times the electronic vector current, with coupling constants $C_{2}$. The former is nuclear spin independent and the latter is nuclear spin dependent.

The Weinberg angle has been accurately determined from a variety of high energy experiments involving the exchange of a $Z^{0}$ between leptons and nuclei; these results are shown in fig. 1 . The 1987 world average value is [3]

$$
\left\langle\sin ^{2} \theta_{w}\right\rangle=0.229 \pm 0.004
$$

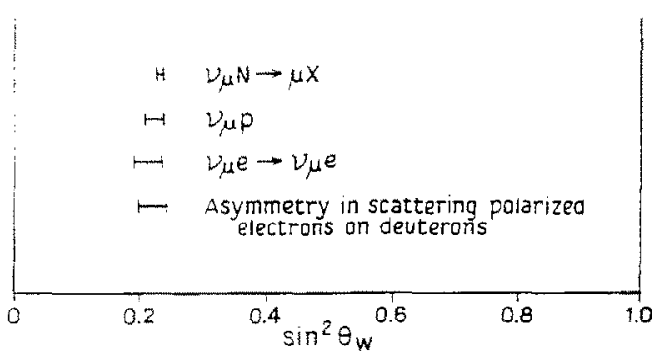

Fig. 1. Values of $\sin ^{2} \theta_{w}$ from high energy experiments. 
There are many experiments which demonstrate the existence of parity nonconservation in heavy atoms ( $\mathrm{Tl}, \mathrm{Bi}, \mathrm{Pb}$ ). The most successful one comes from optical transitions in cesium, as originally predicted by M.A. Bouchiat and C.C. Bouchiat [2]. They proposed looking for the interference between multipole amplitudes of the forbidden optical transition $6 \mathrm{~S} \rightarrow 7 \mathrm{~S}$. The dominant term is either the highly forbidden magnetic dipole moment

$\overrightarrow{\mathrm{M}}=\langle 7 \mathrm{~S}|\vec{\mu}| 6 \mathrm{~S}\rangle$,

or an electric dipole moment which is "Stark-induced" by a static $\boldsymbol{E}$ field, which mixes $7 \mathrm{~S}$ and $7 \mathrm{P}$ states

$\overrightarrow{\mathrm{E}}_{\text {Stark }}=\langle 7 \mathrm{~S}|e E \cdot r| 7 \mathrm{P}\rangle \frac{1}{(\Delta E)}\langle 7 \mathrm{P}|\overrightarrow{e r}| 6 \mathrm{~S}\rangle$.

The parity violation comes from a much smaller electric dipole moment which is "weak-induced", with a weak interaction replacing the Stark interaction,

$\overrightarrow{\mathrm{E}}_{\text {weak }}=\left\langle 7 \mathrm{~S}\left|\mathscr{H}_{\text {weak }}\right| 7 \mathrm{P}\right\rangle \frac{1}{(\Delta E)}\langle 7 \mathrm{P}|\overrightarrow{e r}| 6 \mathrm{~S}\rangle$.

These three terms add coherently to give the total amplitude; the rate includes their interference terms

Transition Rate $\propto\left|\mathrm{M} 1+\mathrm{E} 1_{\text {Stark }}+\mathrm{E} 1_{\text {weak }}\right|^{2}$.

The original idea of using the M1 amplitude proved unworkable; the line was too weak. The E1 $1_{\text {Stark }}$ term gives a transition rate quadratic in the electric field, which can be adjusted to locate and calibrate the line. The interference of $E 1_{\text {Stark }}$ and $E 1_{\text {weak. }}$ gives a term in the event rate linear in the electric field, involving the pseudoscalar $\boldsymbol{P}_{1} \cdot \boldsymbol{P}_{2} \times \boldsymbol{E}$, which changes sign as the configuration is changed from "right-handed" to "lefthanded". Here $\boldsymbol{P}_{1,2}$ stands for the circular polarization vectors of the incident laser light and the outgoing fluorescence light. If a magnetic field is added, there is also a pseudoscalar $\boldsymbol{P}_{1}, \boldsymbol{E} \times \boldsymbol{B}$. The appearance of such pseudoscalar terms is evidence for parity nonconservation, which has been clearly demonstrated in this cesium transition.

The results of more than a decade of experimental work in Paris [4] and Boulder [5] give accurate determinations of the weak-induced dipole moment

$\operatorname{Im} E 1_{\text {weak }}=(-0.79 \pm 0.10) \times 10^{-11} e a_{0} \quad$ (Paris)

Im $\mathrm{E} 1_{\text {weak }}=(-0.83 \pm 0.02) \times 10^{-11} e a_{0} \quad$ (Boulder).

The progress toward this precision is shown in fig. 2 . Comparison of these results with atomic structure calculations gives a value of $\theta_{\mathrm{W}}$ derived from atomic physics [6]

$\sin ^{2} \theta_{\mathrm{W}}(\mathrm{Cs})=0.222 \pm 0.03$

which is in excellent agreement with the world average. The cesium experiments, together with high energy electron-deuteron scattering, provide an accurate confirma-

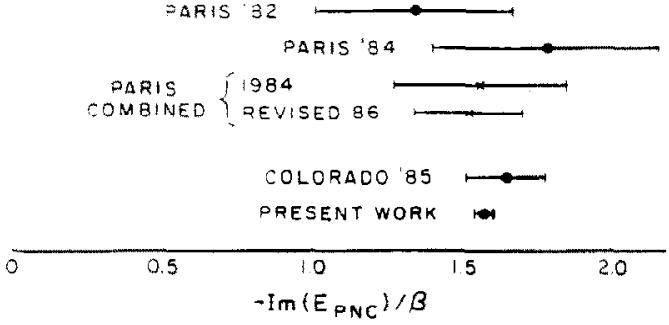

Fig. 2. Progress in measuring $\mathrm{E} 1_{\text {weak }}(\mathrm{Cs})$.

tion over a large range of energy, of the semileptonic coupling constants predicted by the standard model

$C_{1 \mathrm{p}}=-\left(1-4 \sin ^{2} \theta_{\mathrm{W}}\right) / 2 \cong-0.04, \quad C_{1_{\mathrm{n}}}=+1 / 2$.

These coefficients govern the strength of the nuclear spin independent interaction between electrons and nucleons; the coupling of electrons to neutrons is seen to dominate.

The choice of heavy atoms was based mainly on the strong enhancement of the weak-induced dipole moments resulting from the Coulomb attraction of electrons to the nucleus $\left(\propto Z^{2}\right)$ and from the superposition of the "weak charges" of a large number of nucleons, $Q=2\left(Z C_{1 \mathrm{p}}+N C_{1 \mathrm{n}}\right) \propto Z$. This $Z^{3}$ enhancement increases the weak-induced dipole moment in cesium by about $10^{5}$ and plays an important role in the success of the experiments. An equally important role is played by the accurate calculation of the atomic structure factors in $\mathrm{E} 1_{\text {weak }}$; cesium was chosen for its relatively simple structure. The future goal of this work [6] is to increase the precision of both experiment and theory to the $1 \%$ level, in hopes of seeing radiative corrections to the standard model, or "nonstandard" contributions.

The same experiments give evidence for a nuclear spin dependent weak interaction, by comparing $\mathrm{E} 1_{\text {weak }}$ for different hyperfine components of the $6 \mathrm{~S} \rightarrow 7 \mathrm{~S}$ transition. The latest results [5] show that the individual hyperfine transitions give

$$
\begin{aligned}
& \text { Im } \mathrm{E} 1_{\text {weak }}=(-0.861 \pm 0.03) \times 10^{-11} e a_{0} \\
& \quad(F=4 \rightarrow 3), \\
& \operatorname{Im~} \mathrm{E} 1_{\text {weak }}=(-0.794 \pm 0.03) \times 10^{-11} e a_{0} \\
& \quad(F=3 \rightarrow 4) .
\end{aligned}
$$

The differences in these dipole moments are about 12 times smaller than the average dipole moments; the differences also have much less statistical significance (about 2o).

The standard model predicts such an effect via semileptonic interactions, but with small coupling constants

$C_{2 \mathrm{p}}=-C_{2 \mathrm{n}}=-0.62\left(1-4 \sin ^{2} \theta_{W}\right) \cong-0.05$. 
This interaction is also enhanced in heavy atoms by Coulomb effects but not by the superposition of nucleon spins, which pair off rather than adding. The result is a weak-induced dipole moment in cesium smaller by a factor of about 100 , not 12 . There is clearly something clse going on!

The same effect can arise from parity nonconserving hadronic weak interactions within the nucleus, involving $Z^{0}$ exchange between nucleons, together with ordinary photon exchange to the electrons [7]. The (vector) electromagnetic current of a nucleus acquires an axial vector part, through hadronic weak interactions. The dominant effect at low energy is an additional multipole moment $a$, called the "anapole moment" of the nucleus, which produces a magnetic potential

$A(r)=a I \delta(r)$

This relation is obviously parity nonconserving, since $A$ is a true vector and $I$ is a pseudovector. This vector potential gives rise to a contact interaction with the electrons having exactly the same form as $\mathscr{H}_{\mathrm{AV}}$, with coupling strength $C_{\mathrm{A}}$. This coupling constant is defined in the same way as $C_{2}$ and simply adds to it. The anapole moment of cesium has been estimated [8] to have a coupling strength $C_{\mathrm{A}} \equiv 0.3$, about six times larger than $C_{2 \mathrm{p}}$. Hadronic terms are therefore predicted to dominate the semileptonic terms in the spin dependent interaction. This estimate is based on a single particle model of the nucleus and is quite uncertain. For example, it includes only neutral hadron currents and omits the role of charged hadron currents; both should contributc. It is possible to separate the effects of $C_{2}$ from $C_{\mathrm{A}}$, for example by comparison of nuclear isotopes, but this will clearly take more work.

Finally, we mention another possible goal of experiments with heavy atoms: observation of the leptonic interaction, involving $Z^{0}$ exchange between electrons. This makes a negligible contribution to the $7 \mathrm{~S}-7 \mathrm{P}$ mixing in cesium. Parity mixing of two closely spaced $6 \mathrm{p}-5 \mathrm{~d}$ states in samarium has been calculated as an example in which $\mathrm{e}-\mathrm{e}$ interactions might dominate $\mathrm{e}-\mathbf{n}$ interactions [9]. In the central field approximation, these two states should not mix via semileptonic interactions with the nucleus, since the overlap of $5 \mathrm{~d}$ states with the nucleus is suppressed by the angular momentum barrier. However, it was found that the configuration mixing resulting from residual e-e Coulomb interactions gave a semileptonic matrix element larger than the purely leptonic matrix element by about 4000 . Because of the "accidental degeneracy" of these two states, the parity mixing is quite large, but is not due to e-e weak interactions. It appears that parity mixing in heavy atoms is insensitive to the purely leptonic weak interaction.

\section{Parity tests in the hydrogen atom}

There has been a substantial effort to observe parity mixing in the hydrogen atom, but with much less success. The motive for this effort was to test the standard model in an atom for which there is essentially zero theoretical error [10]; hydrogen has always been viewed as the ideal testing ground for fundamental theories.

The experiments were again based on the interference of Stark- and weak-induced electric dipole moments, between two metastable $2 \mathrm{~S}$ states. These transitions are at microwave frequencies, not optical. The $Z^{3}$ enhancement of the weak matrix element is now missing, but is replaced by a reduction of the energy denominators; the $2 \mathbf{S - 2 P}$ states are separated only by the Lamb shift. By applying a magnetic field of about $570 \mathrm{G}$, adjusted to a $2 \mathrm{~S}-2 \mathrm{P}$ level crossing, the energy difference can be further reduced to the $2 P$ level width $\Gamma(2 \mathrm{P}) / 2 \cong 50 \mathrm{MHz}$, which is about $10^{-8}$ atomic units. Another advantage was the clear separation of the effects of $C_{1 p}$ from $C_{2 p}$; state selection makes it possible to measure $C_{2 p}$ alone.

Experiments were begun at three laboratories (Michigan, Seattle and Yale) but after more than a decade of effort have yielded disappointing results. An upper limit $C_{2 p}<300$ has been reported by the Yale group [11] and $C_{2 p}<620$ by Michigan [12]. The principal problems came from systematic effects due to stray electric fields.

\section{Lessons from atomic physics}

In the earliest planning of these experiments, the strategy was dominated by the idea of maximizing the asymmetry, given by the ratio of the two interfering amplitudes, $\mathrm{E} 1_{\text {weak }} / \mathrm{M} 1$ or $\mathrm{E} 1_{\text {weak }} / \mathrm{El}_{\text {Stark }}$. Thus the suppression of M1 transitions was an advantage, as was the ability to adjust $\mathrm{E}_{\text {weak }}$ by the electric field.

The experiment does not necessarily get easier by reducing $\mathrm{M1}$ or $\mathrm{E}$, because the event rate would go down quadratically with these quantities. The signal-tom noise ratio would be independent of $\mathrm{M} 1$ or $\mathrm{E}$. For the hydrogen experiments, it was shown that the optimum signal to noise ratio is given by a simple formula [13]

$(S / N)_{\max } \leq\left\langle 2 S\left|\mathscr{H}_{\text {weak }}\right| 2 \mathbf{P}\right\rangle \sqrt{\tau_{1} \tau_{2}}$,

where $\tau_{1}$ is the time of flight through the interaction region, and $\tau_{2}$ the $2 \mathrm{P}$ lifetime. This result (eq. (1)) is independent of all the applied fields, and is not enhanced by choosing a level crossing! The implication is that, in terms of signal to noise considerations, there is no advantage in a large asymmetry or small energy spacing. There is an advantage in enhancing the weak matrix elements and in long interaction times. 
The final, and for some experiments the fatal, lesson was that systematic errors could swamp the signal. Observation of an asymmetry as "big" as $10^{-5}$ or $10^{-6}$ requires elaborate signal averaging and a high degree of control over, for example, stray electric fields. The best way of expressing the lessons from atomic parity experiments is to downplay the use of any single criterion, and to emphasize the need for satisfying all these criteria. Having a large asymmetry, good signal to noise and control of the systematics are all vital to success.

\section{Parity experiments with ions}

There are NO parity experiments with trapped ions! There are some obvious potential advantages coming from the long "transit time" and the high spatial resolution of a trap, but there is a big disadvantage in the relatively small number of ions which can be stored. Only one experiment has been described in the literature [14]; it was designed as a measurement of $C_{2}$ through parity mixing of the ground state hyperfine multiplets in singly charged ions. It was never completed.

Many people have considered parity experiments in highly charged ions from accelerators [15,16]. These ions can offer both the advantages of enhanced matrix elements and of calculable atomic structures. Hydrogenic ions have the added feature of closely spaced levels with opposite parity. But the advantages seem to lie in $\mathrm{He}^{+}$, at low $Z$ rather than at high $Z$ [17].

The reasons for this can be illustrated with the one-photon decay of the metastable state, $2 S \rightarrow 1 S+\gamma$. The spontaneous M1 moment scales like $Z^{2}$, and the weak-induced E1 like $Q Z^{3} / S$. The enhancement of the weak matrix element $\left(Q Z^{4}\right)$ is even stronger than in heavy atoms. This is compensated by the decrease in electric dipole moments and by the rapid growth of the energy denominator (Lamb shift) $S$, which scales like $Z^{4} F(Z)$. The result is a decay asymmetry $\hat{\boldsymbol{P}} \cdot \hat{\boldsymbol{k}}$ for polarized ions which decreases strongly with increasing $Z$ :

$$
\text { Asymmetry }=\mathrm{E} 1 / \mathrm{M} 1=\left(\sqrt{2} G_{F} Q\right) / \alpha(\alpha Z)^{3} F(Z) .
$$

The signal to noise ratio increases slowly for large $Z$, because the hranching ratio for single photon decay grows like $Z^{10}$. Thus the two criteria are mutually exclusive and cannot both be satisfied. There are several other asymmetries to consider, but this one is the most favorable in hydrogenic ions.

The case of one-photon decays in He-like ions has also been considered [18], especially near the accidental degeneracy of $2^{1} \mathrm{~S}_{0}$ and $2{ }^{3} \mathrm{P}_{1}$ which occurs near $Z=6$.
The weak-induced E1 is enhanced both by Coulomb effects and by small energy denominators, and interferes with the M1 moment which comes from hyperfine mixing. The maximum asymmetry at $Z=6$ is about $10^{-4}$. The branching ratio for this $\mathrm{M} 1$ transition grows strongly with $\mathcal{Z}$, making the signal-to-noise ratio hopelessly small for those ions near the level crossing. Again, these two criteria are in conflict.

In retrospect, the advantages of close energy spacing and the availability of accurate atomic structure calculations in hydrogenic ions are overemphasized; these features are not limited to one-electron ions. Calculations can be done in other highly charged ions to the required accuracy (1\%). Remeasuring $Q$ is not a good idea either; experiments with highly charged ions would be more worthwhile if they could solve the problems which remain unresolved with atoms.

One suggestion is to re-examine the e-e interaction in ions. As $Z$ increases, the contribution of central forces becomes larger relative to residual interactions. One should reconsider the calculations of ref. [10] with ions, near an "accidental" degeneracy of higher angular momentum states. One hopes to find a case where weak matrix elements are enhanced, energy spacings reduced and central forces dominant, in a highly charged ion.

Another goal might be to search for nuclear spin dependent effects in ions. For example, one could use ground state hyperfine transitions for this purpose, since these states are stable and may permit much longer "transit times". Since these transitions involve flipping the nuclear spin, they are proportional to $C_{2}$ [19]. Such transitions in heavy ions are shifted to optical frequencies. However, it appears that the Coulomb enhancement of the weak matrix element in hydrogenic ions grows only linearly in $Z$ and remains smaller than in heavy neutral atoms, where they exhibit a $Z^{2}$ enhancement [20].

\section{Conclusions}

There is good evidence of nuclear spin independent terms in cesium, consistent with the standard model of semileptonic weak interactions. There is some evidence in cesium of nuclear spin dependent terms, probably dominated by hadronic weak interactions. There is no evidence of purely leptonic weak interactions.

One- and two-electron ions do not seem to provide hope of parity experiments. The best strategy for new experiments with highly charged ions is probably to search for an ion with some accidental degeneracy, especially higher angular momentum states which may be sensitive to the $\mathrm{e}-\mathrm{e}$ weak interaction. 


\section{References}

[1] B.W. Lynn, in: Atomic Physics 9, eds. R.S. Van Dyck and E.N. Fortson (World Scientific, Singapore, 1984) p. 212.

[2] M.A. Bouchiat and C.C. Bouchiat, Phys. Lett. B48 (1974) 111.

[3] U. Amaldi et al., Phys. Rev. D36 (1987) 1385.

[4] M.A. Bouchiat et al., J. Phys. (Paris) 47 (1986) 1709.

[5] C. Noecker et al., Phys. Rev. Lett. 61 (1988) 310.

[6] C.A. Piketty, 23rd Rencontres de Moriond, Electroweak Interactions, Les Arcs (1988).

[7] V.V. Flambaum et al., Phys. Lett. B146 (1984) 367.

[8] P.A. Frantsuzov and I.B. Khriplovich, Z. Phys. D7 (1988) 297.

[9] M.A. Gongora and P.G.H. Sandars, J. Phys. BL291 (1486).

[10] R.W. Dunford et al., Phys. Rev. A18 (1978) 242.

[11] E.A. Hinds, The Hydrogen Atom, eds. G.F. Bassani, M. Inguscio and T.W. Hänsch (Springer, Berlin, 1989) p. 268.
[12] L.P. Levy and W.L. Williams, Phys. Rev, Lett. 48 (1982) 607.

[13] E.A. Hinds, Phys. Rev. Lett. 44 (1980) 374.

[14] G. Gräff, Z. Phys. A305 (1982) 107.

[15] E.G. Myers, First Year Essay, Oxford Univ. preprint (1979).

[16] R.W. Dunford and R.R. Lewis, Phys. Rev. A23 (1981) 10.

[17] R.W. Dunford, Princeton Univ, preprint (1985).

[18] V.G. Gorshkov and L.N. Labzovskii, Sov. Phys. JETP 42 (1976) 581.

[19] V.N. Novikov and I.B. Khriplovich, JETP Lett. 22 (1975) 74 ;

C.E. Loving and P.G.H. Sandars, J. Phys. B10 (1977) 2755.

[20] M. Sah, PhD dissertation, Univ. of Michigan (1987). 